\title{
Improved Linear Parallel Interference Cancellers
}

\author{
T. Srikanth, K. Vishnu Vardhan, A. Chockalingam, Senior Member, IEEE, and L. B. Milstein, Fellow, IEEE
}

\begin{abstract}
In this paper, taking the view that a linear parallel interference canceller (LPIC) can be seen as a linear matrix filter, we propose new linear matrix filters that can result in improved bit error performance compared to other LPICs in the literature. The motivation for the proposed filters arises from the possibility of avoiding the generation of certain interference and noise terms in a given stage that would have been present in a conventional LPIC (CLPIC). In the proposed filters, we achieve such avoidance of the generation of interference and noise terms in a given stage by simply making the diagonal elements of a certain matrix in that stage equal to zero. Hence, the proposed filters do not require additional complexity compared to the CLPIC, and they can allow achieving a certain error performance using fewer LPIC stages. We also extend the proposed matrix filter solutions to a multicarrier DS-CDMA system, where we consider two types of receivers. In one receiver (referred to as Type-I receiver), LPIC is performed on each subcarrier first, followed by multicarrier combining (MCC). In the other receiver (called Type-II receiver), MCC is performed first, followed by LPIC. We show that in both Type-I and Type-II receivers, the proposed matrix filters outperform other matrix filters. Also, Type-II receiver performs better than Type-I receiver because of enhanced accuracy of the interference estimates achieved due to frequency diversity offered by MCC.
\end{abstract}

Index Terms-Linear parallel interference cancellation, linear matrix filters, decorrelating detector, MMSE detector, multicarrier DS-CDMA.

\section{INTRODUCTION}

$\mathbf{L}$ INEAR parallel interference cancellers (LPIC) have the advantages of implementation simplicity, analytical tractability, and good performance [1]-[12]. The conventional way to realize LPIC schemes is to use unscaled values of the previous stage soft outputs of different users for multiple access interference (MAI) estimation. In [3], Guo et al described and analyzed LPIC schemes for CDMA using a matrix-algebraic approach. They pointed out that an LPIC can be viewed as a linear matrix filter applied directly to the chip matched filter (MF) output vector. While the matrix filter

Manuscript received March 29, 2007; revised July 16, 2007; accepted September 12, 2007. The associate editor coordinating the review of this paper and approving it for publication was D. Hong. This work in part was presented in the IEEE International Conference on Communications, Glasgow, June 2007, and in the National Conference on Communications, Indian Institute of Technology, Kanpur, India, January 2007. This work was supported in part by the Air Force Office of Scientific Research under grant \# FA9550-06-1-0210.

T. Srikanth is with ORCA Radio Systems Private Limited, Bangalore 560095, India (e-mail: thatisrikanth@ymail.com).

K. Vishnu Vardhan is with Cisco Systems (India) Private Limited, Bangalore 560087, India (e-mail: vishnu.pgece4miisc@gmail.com).

A. Chockalingam is with the Department of Electrical Communication Engineering, Indian Institute of Science, Bangalore 560012, India (e-mail: achockal@ece.iisc.ernet.in).

L. B. Milstein is with the Department of Electrical and Computer Engineering, University of California, San Diego, La Jolla, CA 92093, USA (e-mail: milstein@ece.ucsd.edu).

Digital Object Identifier 10.1109/TWC.2008.070332. corresponding to the conventional LPIC (CLPIC) converges to the decorrelating (DC) detector, they also proposed a modified matrix filter which converges to a minimum mean square error (MMSE) detector. This was done by exploiting the equivalence of the LPIC to a steepest descent optimization method for minimizing the mean square error. For this optimization, they obtained optimum step sizes for different stages that remove the excess mean square error in $K$ stages (where $K$ is the number of users), leaving only the minimum MSE in stages greater than $K$. The condition for this convergence has been shown to be that the maximum eigenvalue of the correlation matrix must be less than two.

Our contribution in this paper is that we propose new linear matrix filters that can perform better than the matrix filters studied in [3]. The motivation for the proposed filters arises from the possibility of avoiding the generation of certain interference and noise terms in a given stage that would have been present in the CLPIC. In the proposed filters, we achieve such avoidance of the generation of interference and noise terms in a given stage by simply making the diagonal elements of a certain matrix in that stage equal to zero. Hence the proposed filters do not require additional complexity compared to the CLPIC. We show that the proposed matrix filters can achieve better performance compared to other matrix filters in the literature. This, in turn, can allow achieving a certain error performance using fewer LPIC stages. We also propose filters that use different step sizes for different stages (but the same step size for all users at a given stage). In addition, we propose filters that use different weights for different users in different stages, where we also obtain closed-form expressions for the optimum weights that maximize the output average SINR in a given stage.

We further extend the proposed matrix filter solutions to a multicarrier DS-CDMA system, where multicarrier combining (MCC) needs to be carried out in addition to the LPIC operation. Depending on which operation (i.e., MCC or LPIC) gets done first at the receiver, the resulting performances and complexities differ. We consider two types of receivers. In what we call the Type-I receiver, LPIC is performed on each subcarrier first, followed by MCC [12]. In the Type-II receiver, MCC is performed first, followed by LPIC. We show that in both Type-I and Type-II receivers, the proposed matrix filters outperform other matrix filters. Also, Type-II receiver outperforms Type-I receiver because of enhanced accuracy of the MAI estimates achieved due to frequency diversity offered by MCC.

The rest of the paper is organized as follows. In Sec. II, we present the system model. In Sec. III, we present the proposed matrix filters for single carrier DS-CDMA, along with their bit error performance results. Section IV presents 
the proposed filters and their performance in multicarrier DSCDMA. Conclusions are presented in Sec. V.

\section{SySTEM MODEL}

We consider a $K$-user synchronous multicarrier DS-CDMA system with $M$ subcarriers. Let $b_{k} \in\{+1,-1\}$ denote the binary data symbol of the $k$ th user, which is sent in parallel on $M$ subcarriers [13]. Let $P$ denote the number of chipsper-bit in the signature waveforms. It is assumed that the channel is frequency non-selective on each subcarrier and the fading is slow (assumed constant over one bit interval) and independent from one subcarrier to the other. We assume that the subcarriers are separated sufficiently apart so that intercarrier interference is negligible.

Let $\mathbf{y}^{(1),(i)}=\left[\begin{array}{llll}y_{1}^{(1),(i)} & y_{2}^{(1),(i)} & \cdots & y_{K}^{(1),(i)}\end{array}\right]^{T}$ denote the $K$ length received signal vector ${ }^{1}$ at the MF output ${ }^{2}$ on the $i$ th subcarrier; i.e., $y_{k}^{(1),(i)}$ is the MF output (i.e., 1st stage output) of the $k$ th user on the $i$ th subcarrier, given by

$$
y_{k}^{(1),(i)}=\underbrace{A_{k} b_{k} h_{k}^{(i)}}_{\text {desired signal }}+\underbrace{\sum_{j=1, j \neq k}^{K} \rho_{k j}^{(i)} A_{j} b_{j} h_{j}^{(i)}}_{\text {MAI }}+\underbrace{n_{k}^{(i)}}_{\text {noise }} .
$$

The MF output vector $\mathbf{y}^{(1),(i)}$ can be written in the form

$$
\mathbf{y}^{(1),(i)}=\mathbf{R}^{(i)} \mathbf{H}^{(i)} \mathbf{b}+\mathbf{n}^{(i)},
$$

where $\mathbf{H}^{(i)}$ represents the $K \times K$ channel matrix, given by

$$
\mathbf{H}^{(i)}=\operatorname{diag}\left\{h_{1}^{(i)}, h_{2}^{(i)}, \cdots, h_{K}^{(i)}\right\},
$$

and the channel coefficients $h_{k}^{(i)}, i=1,2, \cdots, M$, are assumed to be i.i.d. complex Gaussian r.v's with zero mean and $E\left[\left(\operatorname{Re}\left\{h_{k}^{(i)}\right\}\right)^{2}\right]=E\left[\left(\operatorname{Im}\left\{h_{k}^{(i)}\right\}\right)^{2}\right]=0.5$. The matrix $\mathbf{R}^{(i)}$ is the $K \times K$ cross-correlation matrix on the $i$ th subcarrier, given by

$$
\mathbf{R}^{(i)}=\left[\begin{array}{cccc}
1 & \rho_{12}^{(i)} & \cdots & \rho_{1 K}^{(i)} \\
\rho_{21}^{(i)} & 1 & \cdots & \rho_{2 K}^{(i)} \\
\vdots & \vdots & \ddots & \vdots \\
\rho_{K 1}^{(i)} & \rho_{K 2}^{(i)} & \cdots & 1
\end{array}\right],
$$

where $\rho_{l j}^{(i)}=\rho_{j l}^{(i)}$ is the normalized cross-correlation coefficient between the signature waveforms of the $l$ th and $j$ th users on the $i$ th subcarrier. The $K$-length data vector $\mathbf{b}$ is given by $\mathbf{b}=\left[\begin{array}{llll}A_{1} b_{1} & A_{2} b_{2} & \cdots & A_{K} b_{K}\end{array}\right]^{T}$, where $A_{k}$ denotes the transmit amplitude of the $k$ th user. The $K$ length noise vector $\mathbf{n}^{(i)}$ on the $i$ th subcarrier is given by $\mathbf{n}^{(i)}=\left[\begin{array}{llll}n_{1}^{(i)} & n_{2}^{(i)} & \cdots & n_{K}^{(i)}\end{array}\right]^{T}$, which is assumed to be complex Gaussian with zero mean with $E\left[n_{k}^{(i)}\left(n_{j}^{(i)}\right)^{*}\right]=\sigma^{2}$ when $j=k$ and $E\left[n_{k}^{(i)}\left(n_{j}^{(i)}\right)^{*}\right]=\sigma^{2} \rho_{k j}^{(i)}$ when $j \neq k$.

\footnotetext{
${ }^{1}$ Vectors are denoted by boldface lowercase letters, and matrices are denoted by boldface uppercase letters. [.] $]^{T}$ and [.] ${ }^{H}$ denote transpose and conjugate transpose operations, respectively. $\operatorname{Re}\{\alpha\}$ and $\operatorname{Im}\{\alpha\}$ denote the real and imaginary parts of $\alpha$.

${ }^{2}$ We take the MF output (i.e., the despread output) as the 1st stage output in the multistage LPIC receivers. So, the ${ }^{(m),(i)}$ in the superscript of $y$ denotes the stage index $m$ and subcarrier index $i$.
}

\section{Proposed Linear Matrix Filters}

In this section, we propose improved LPICs for single carrier DS-CDMA (i.e., $M=1$ in the system model presented in Sec. II). So, for notational simplicity, we drop the subcarrier index $(i)$ in this section. In Sec. IV, we will consider $M>1$. We assume that all the channel coefficients are perfectly known at the receiver. Dropping the subcarrier index in (1), the MF output of the desired user $k, y_{k}^{(1)}$, can be written as

$$
y_{k}^{(1)}=\underbrace{x_{k}}_{\text {desired signal }}+\underbrace{\sum_{j=1, j \neq k}^{K} x_{j} \rho_{k j}}_{\text {MAI }}+\underbrace{n_{k}}_{\text {noise }},
$$

where $x_{l} \triangleq A_{l} b_{l} h_{l}, l=1,2, \cdots, K$.

\section{A. Conventional Matrix Filter, $\mathbf{G}^{(m)}$}

In conventional LPIC (CLPIC), an estimate of the MAI for the desired user in the current stage is obtained using all the other users' soft outputs from the previous stage for cancellation in the current stage. The $m$ th stage output of the desired user $k, y_{k}^{(m)}$, in CLPIC is [9]

$$
y_{k}^{(m)}=y_{k}^{(1)}-\underbrace{\sum_{j=1, j \neq k}^{K} \rho_{j k} y_{j}^{(m-1)}}_{\text {MAI estimate }} .
$$

The $k$ th user's bit decision after MAI cancellation in the $m$ th stage, $\widehat{b}_{k}^{(m)}$, is obtained as

$$
\widehat{b}_{k}^{(m)}=\operatorname{sgn}\left(\operatorname{Re}\left(h_{k}^{*} y_{k}^{(m)}\right)\right) .
$$

The CLPIC output in (6) can be written in matrix algebraic form as [3]

$$
\begin{aligned}
\mathbf{y}^{(m)} & =\left(\mathbf{I}+(\mathbf{I}-\mathbf{R})+\cdots+(\mathbf{I}-\mathbf{R})^{m-1}\right) \mathbf{y}^{(1)} \\
& =\underbrace{\sum_{j=1}^{m}(\mathbf{I}-\mathbf{R})^{j-1}}_{\mathbf{G}^{(m)}} \mathbf{y}^{(1)} .
\end{aligned}
$$

The $\mathbf{G}^{(m)}$ filter in (8) can be viewed as an equivalent one-shot linear matrix filter for the $m$ th stage of the CLPIC.

\section{B. Proposed Matrix Filter, $\mathbf{G}_{\mathbf{p}}{ }^{(m)}$}

In this subsection, we propose a new linear matrix filter, which we denote as $\mathbf{G}_{\mathbf{p}}{ }^{(m)}$, that can perform better than the matrix filter $\mathbf{G}^{(m)}$ in (8). The motivation for the new matrix filter can be explained as follows.

What does the matrix filter $\mathbf{G}^{(m)}$ do: It is noted that the behavior of the $\mathbf{G}^{(m)}$ filter in (8) (i.e., CLPIC) at a given stage $m \geq 2$ is characterized by $a$ ) interference removal, $b)$ generation of new interference terms, $c$ ) desired signal loss/gain, $d$ ) desired signal recovery/removal, and e) noise enhancement. For example, the cancellation operation in the 2nd stage (i.e., $m=2$ ) results in $i$ ) interference removal, $i i$ ) generation of new interference terms, iii) desired signal loss, and $i v)$ noise enhancement. This can be seen by observing 
the 2nd stage output expression for the desired user $k$, which can be written, using (6) and (5), as

$$
\begin{aligned}
y_{k}^{(2)}= & y_{k}^{(1)}-\sum_{j=1, j \neq k}^{K} \rho_{j k} y_{j}^{(1)} \\
= & \left(x_{k}+\sum_{i=1, i \neq k}^{\sum_{k}} \rho_{k i} x_{i}+n_{k}\right) \\
& -\sum_{j=1, j \neq k}^{K} \rho_{j k}(x_{j}+\underbrace{\left.\sum_{1=1 \neq j}^{K} \rho_{j l} x_{l}+n_{j}\right)}_{l \text { can be } k \text { here }} \\
= & x_{k}-\underbrace{x_{k} \sum_{j=1, j \neq k}^{\sum_{j k}^{K}} \rho_{\text {new interference terms }}^{\sum_{j=1, j \neq k}^{K} \rho_{j k}} \sum_{l=1, l \neq j, k}^{K} \rho_{j l} x_{l}}_{\text {desired signal loss }} \\
& +n_{k}-\underbrace{\sum_{j=1, j \neq k}}_{\sum_{\text {additional noise terms }}^{K} \rho_{j k} n_{j}}
\end{aligned}
$$

Comparing the expression at the MF output, $y_{k}^{(1)}$, in (5) and the expression for the 2nd stage output, $y_{k}^{(2)}$, in (9), it can be seen that the cancellation operation in the 2 nd stage results in the following at the 2 nd stage output.

- The interference terms, $\sum_{j \neq k} \rho_{j k} x_{j}$, that were present in the MF output in (5) are removed. In the process, $i$ ) new interference terms proportional to $\rho^{2}$, i.e., $\sum_{j \neq k} \rho_{j k} \sum_{l \neq j, k} \rho_{j l} x_{l}$ in (9), get generated, $\left.i i\right)$ a fraction $\sum_{j \neq k} \rho_{j k}^{2}$ of the desired signal component gets lost,

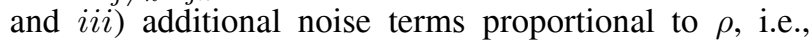
$\sum_{j \neq k} \rho_{j k} n_{j}$ in (9), get introduced.

In Appendix A, we present the expression for the 3rd stage output in an expanded form. From (44) in Appendix A, we can make the following observations which result from the cancellation operation in the 3rd stage.

- The desired signal loss that occurred in the 2nd stage is recovered (see the two $A$ terms cancelling each other in (44)). In the process, new interference terms proportional to $\rho^{3}$ (see the $B_{I}$ term in (44)) as well as additional noise terms proportional to $\rho^{2}$ (see the $B_{N}$ term in (44)) get generated.

- Interference terms generated in the 2 nd stage are removed (see the two $C$ terms cancelling each other in (44)). In the process, $i$ ) further desired signal loss/gain ${ }^{3}$ proportional to $\rho^{3}$ occurs (see the $D$ term in (44)), and $i i$ ) new interference terms proportional to $\rho^{3}$ (see the $E_{I}$ term in (44)) as well as additional noise terms proportional to $\rho^{2}$ (see the $E_{N}$ term in (44)) get generated.

Similar observations can be made on the expanded form of the equations for the subsequent stages of the CLPIC ${ }^{4}$. For $m \rightarrow \infty$, the CLPIC is known to converge to the decorrelating

\footnotetext{
${ }^{3}$ Depending on $\rho$ 's being positive or negative, the term $D$ in (44) can be positive or negative, because of which there can be a desired signal gain or loss.

${ }^{4}$ The general expression for the $m$ th stage output in expanded form, for any $m \geq 3$, and the corresponding observations are given in Appendix B.
}

detector, provided the eigenvalues of the $\mathbf{R}$ matrix are less than two [3]. That is, when $m \rightarrow \infty$, in the expression for $\mathbf{G}^{(m)}$ output in (48), the desired signal loss/gain and the interference terms go to zero and the noise term gets enhanced.

Proposed matrix filter, $\mathbf{G}_{\mathbf{p}}{ }^{(m)}$ : We propose to avoid the generation of new interference and noise terms in $T_{3}$ in (47), caused in the process of recovery/removal of desired signal loss/gain in the previous stage. Since there is no desired signal loss/gain in the 1st stage, the 2nd stage of the proposed filter is the same as that of the $\mathbf{G}^{(m)}$ filter, i.e., $\mathbf{G}_{\mathbf{p}}{ }^{(2)}=\mathbf{G}^{(2)}$. For stages greater than two, i.e., for $m \geq 3$, the $m$ th stage output of the proposed filter $\mathbf{G}_{\mathbf{p}}{ }^{(m)}$, denoted by $y_{k, \mathbf{p}}^{(m)}$, can be written as

$$
\begin{gathered}
y_{k, \mathbf{p}}^{(m)}=y_{k, \mathbf{p}}^{(m-1)}+(-1)^{m+1} \sum_{k_{1} \neq k}^{K} \sum_{k_{2} \neq k, k_{1}}^{K} \sum_{k_{3} \neq k, k_{2}}^{K} \cdots \\
\sum_{k_{m-2} \neq k, k_{m-3}}^{K} \sum_{k_{m-1} \neq k, k_{m-2}}^{K} \rho_{k k_{m-1}} \rho_{k_{m-1} k_{m-2}} \cdots \rho_{k_{2} k_{1}} y_{k_{1}}^{(1)} .
\end{gathered}
$$

We note that the above expression is obtained by $i$ ) dropping $T_{3}$ from (47), and $i i$ ) modifying $T_{4}$ in (47) such that all the summations in it exclude the desired user index $k$. The above two modifications ensure that the proposed filter removes the previous stage interference while avoiding the recovery/removal of the desired signal loss/gain ${ }^{5}$. Also, because of these modifications, the interference and noise terms in a given stage of the proposed filter will be a subset of the interference and noise terms in the same stage of the $\mathbf{G}^{(m)}$ filter. Equation (10) can be written in the following form

$$
\begin{array}{r}
y_{k, \mathbf{p}}^{(m)}=y_{k}^{(1)}-\sum_{k_{1} \neq k}^{K}\left(\rho_{k k_{1}}-\sum_{k_{2} \neq k, k_{1}}^{K} \rho_{k k_{2}} \rho_{k_{2} k_{1}}\right. \\
+\sum_{k_{2} \neq k, k_{1}}^{K} \sum_{k_{3} \neq k, k_{2}}^{K} \rho_{k k_{3}} \rho_{k_{3} k_{2}} \rho_{k_{2} k_{1}}-\cdots \\
\left.+(-1)^{m} \sum_{k_{2} \neq k, k_{1}}^{K} \sum_{k_{3} \neq k, k_{2}}^{K} \ldots \sum_{k_{m-1} \neq k, k_{m-2}}^{K} \rho_{k k_{m-1}} \cdots \rho_{k_{2} k_{1}}\right) y_{k_{1}}^{(1)},
\end{array}
$$

which, in turn, can be expressed in matrix form as

$$
\mathbf{y}_{\mathbf{p}}^{(m)}=\underbrace{\left(\sum_{j=0}^{m-1} \mathbf{B}_{j}\right)}_{\mathbf{G}_{\mathbf{p}}(m)} \mathbf{y}^{(1)},
$$

where

$$
\mathbf{B}_{n}=\left[\mathbf{B}_{n-1}(\mathbf{I}-\mathbf{R})\right]^{\odot},
$$

$[\mathbf{M}]^{\odot}$ denotes the matrix $\mathbf{M}$ with its diagonal elements made equal to zero, and $\mathbf{B}_{0}=\mathbf{I}$. Note that, since (12) is structurally the same as (8) and the $[.]^{\odot}$ operation in (13) does not add to complexity, the proposed $\mathbf{G}_{\mathbf{p}}{ }^{(m)}$ filter has the same complexity as the $\mathbf{G}^{(m)}$ filter.

The $\mathbf{G}^{(m)}$ filter is known to converge to the decorrelating detector for $m \rightarrow \infty$, provided the maximum eigenvalue of

\footnotetext{
${ }^{5}$ Although possible signal loss recovery is avoided in the process, the net effect can still be beneficial (we will see this in Sec. III-F).
} 
the $\mathbf{R}$ matrix is less than two [3]. That is, $\mathbf{G}^{(\infty)}=\mathbf{R}^{-1}$, which results in the output vector

$$
\left(\mathbf{y}^{(\infty)}\right)_{\mathbf{G}}=\mathbf{R}^{-1} \mathbf{y}^{(1)}=\mathbf{x}+\mathbf{R}^{-1} \mathbf{n} .
$$

As with $\mathbf{G}^{(m)}$, all the interference terms in $\mathbf{G}_{\mathbf{p}}{ }^{(m)}$ also go to zero for $m \rightarrow \infty$. This can be seen as follows. From (12) and (13), $\mathbf{G}_{\mathbf{p}}{ }^{(\infty)}$ can be written in the form

$$
\begin{aligned}
\mathbf{G}_{\mathbf{p}}{ }^{(\infty)}= & \underbrace{\mathbf{I}}_{\mathbf{B}_{0}}+\underbrace{\left\{(\mathbf{I}-\mathbf{R})-\mathbf{D}_{1}\right\}}_{\mathbf{B}_{1}} \\
& +\underbrace{\left\{\left\{(\mathbf{I}-\mathbf{R})-\mathbf{D}_{1}\right\}(\mathbf{I}-\mathbf{R})-\mathbf{D}_{2}\right\}}_{\mathbf{B}_{2}}+\cdots,
\end{aligned}
$$

where $\mathbf{D}_{n}$ is a diagonal matrix with the diagonal elements the same as those in the matrix $\mathbf{B}_{n-1}(\mathbf{I}-\mathbf{R})$. Equation (15) can be written as

$$
\mathbf{G}_{\mathbf{p}}{ }^{(\infty)}=\underbrace{\left(\mathbf{I}-\mathbf{D}_{1}-\mathbf{D}_{2}-\cdots\right)}_{\triangleq \mathbf{F}} \mathbf{R}^{-1} .
$$

Hence, the output vector for $m \rightarrow \infty$ is given by

$$
\left(\mathbf{y}^{(\infty)}\right)_{\mathbf{G}_{\mathbf{p}}}=\mathbf{F R}^{-1} \mathbf{y}^{(1)}=\mathbf{F} \mathbf{x}+\mathbf{F R}^{-1} \mathbf{n} .
$$

The diagonal matrix $\mathbf{F}$ defined in (16) can be written as

$$
\mathbf{F}=\operatorname{diag}\left(f_{1}, f_{2}, \cdots, f_{K}\right),
$$

where $f_{k}$ is given by

$$
\begin{aligned}
f_{k}= & 1-\sum_{k_{1} \neq k}^{K} \rho_{k k_{1}} \rho_{k_{1} k}+\sum_{k_{1} \neq k}^{K} \sum_{k_{2} \neq k, k_{1}}^{K} \rho_{k k_{2}} \rho_{k_{2} k_{1}} \rho_{k_{1} k} \\
& -\sum_{k_{1} \neq k}^{K} \sum_{k_{2} \neq k, k_{1}}^{K} \sum_{k_{3} \neq k, k_{2}}^{K} \rho_{k k_{3}} \rho_{k_{3} k_{2}} \rho_{k_{2} k_{1}} \rho_{k_{1} k} \cdots
\end{aligned}
$$

For the case of equi-correlated users, $f_{k}$ in (19) can be shown to converge to $1-\left((K-1) \rho^{2} /(1+(K-2) \rho)\right.$, and there are no interference terms in (17). Also, note that the outputs of the $\mathbf{G}$ filter in (14) and the $\mathbf{G}_{\mathbf{p}}$ filter in (17) have the same SNR for $m \rightarrow \infty$.

\section{Why $\mathbf{G}_{\mathbf{p}}{ }^{(m)}$ can perform better than $\mathbf{G}^{(m)}$ - An Illustration}

To analytically see why $\mathbf{G}_{\mathbf{p}}{ }^{(m)}$ can perform better than $\mathbf{G}^{(m)}$ for $m \geq 3$, consider the case of $K$ equi-correlated users with correlation coefficient $\rho$, and no noise. Let us consider the average signal-to-interference ratio (SIR) at the 3rd stage output for $\mathbf{G}_{\mathbf{p}}{ }^{(m)}$ and $\mathbf{G}^{(m)}$. The 3rd stage output of $\mathbf{G}^{(m)}$, in the absence of noise, can be written as

$$
\left(y_{k}^{(3)}\right)_{\mathbf{G}}=(1+D) x_{k}+B_{I}+E_{I},
$$

where the terms $D, B_{I}, E_{I}$ are defined in (44). Likewise, the 3rd stage output of $\mathbf{G}_{\mathbf{p}}{ }^{(m)}$ can be written as

$$
\left(y_{k}^{(3)}\right)_{\mathbf{G}_{\mathbf{p}}}=(1-A+D) x_{k}+E_{I} .
$$

Note that the interference term $B_{I}$ generated in $\mathbf{G}^{(m)}$ is not generated in $\mathbf{G}_{\mathbf{p}}{ }^{(m)}$. Also, the desired signal term $A$ is recovered in $\mathbf{G}^{(m)}$ whereas it is not recovered in $\mathbf{G}_{\mathbf{p}}{ }^{(m)}$. Now, from (20), the average SIR at the 3rd stage output of $\mathbf{G}^{(m)}$, for the case of equi-correlated and equal-amplitude users, can be obtained as

$$
(\overline{\mathrm{SIR}})_{\mathbf{G}}^{(3)}=\frac{\left(1+(K-1)(K-2) \rho^{3}\right)^{2}}{(K-1)\left((K-1) \rho^{3}+(K-2)^{2} \rho^{3}\right)^{2}} .
$$

Likewise, from (21), the average SIR at the 3rd stage output of $\mathbf{G}_{\mathbf{p}}{ }^{(m)}$ can be obtained as

$$
(\overline{\mathrm{SIR}})_{\mathbf{G}_{\mathbf{p}}}^{(3)}=\frac{\left(1-(K-1) \rho^{2}+(K-1)(K-2) \rho^{3}\right)^{2}}{(K-1)\left((K-2)^{2} \rho^{3}\right)^{2}} .
$$

From (22) and (23), it can be seen that

$$
\beta \triangleq \sqrt{\frac{(\overline{\mathrm{SIR}})_{\mathbf{G}_{\mathbf{p}}}^{(3)}}{(\overline{\mathrm{SIR}})_{\mathbf{G}}^{(3)}}}=1+\delta
$$

where

$$
\delta=\frac{(K-1)(1-(K-2) \rho)\left(1+(K-2) \rho-(K-1) \rho^{2}\right)}{(K-2)^{2}\left(1+(K-1)(K-2) \rho^{3}\right)} .
$$

For $\rho>0$, the maximum eigenvalue for the $\mathbf{R}$ matrix is $1+$ $(K-1) \rho$, so that the condition for convergence is $1+(K-$ 1) $\rho<2$, i.e., $(K-1) \rho<1$. Now, in (24), the 2 nd term is positive when $(K-1) \rho<1$, which results in $\beta>1$. This implies that $\mathbf{G}_{\mathbf{p}}{ }^{(m)}$ results in a higher average output SIR than $\mathbf{G}^{(m)}$. As will be seen in Sec. III-F, simulation results show that $\mathbf{G}_{\mathbf{p}}{ }^{(m)}$ can perform better than $\mathbf{G}^{(m)}$ in the case of non-equicorrelated users as well as in the presence of noise.

\section{A Modified MMSE Converging Filter, $\mathbf{G}_{\mathbf{p} \mu}{ }^{(m)}$}

As pointed out in Sec. I, Guo et al, in [3], have proposed modifications to the $\mathbf{G}^{(m)}$ filter so that the resulting modified matrix filter converges to the MMSE detector instead of the decorrelating detector, by exploiting the equivalence of the LPIC to the steepest descent method (SDM) of optimization for minimizing the MSE. They also derived optimum step sizes for various stages, which ensured convergence to the MMSE detector in $K$ stages, where $K$ is the number of users. We refer to this MMSE converging matrix filter proposed by Guo et al in [3] as the $\mathbf{G}_{\mu}{ }^{(m)}$ filter, which is given by [3]

$$
\mathbf{y}_{\mu}^{(m)}=\mathbf{G}_{\mu}{ }^{(m)} \mathbf{y}^{(1)},
$$

where

$$
\mathbf{G}_{\mu}{ }^{(m)}=\mu_{m} \mathbf{I}+\sum_{i=1}^{m-1} \mu_{m-i} \prod_{j=1}^{i}\left(\mathbf{I}-\mu_{m-i+j}\left(\mathbf{R}+\sigma^{2} \mathbf{I}\right)\right),
$$

$\mu_{i}$ is the step size at stage $i$, the optimum values of which were obtained to be

$$
\mu_{i}=\frac{1}{\lambda_{i}+\sigma^{2}}, \quad i=1,2, \cdots, K,
$$

where $\lambda_{i}, i=1,2, \cdots, K$ are eigenvalues of matrix $\mathbf{R}$. We note that a similar SDM view can be taken to modify our proposed matrix filter $\mathbf{G}_{\mathbf{p}}{ }^{(m)}$ so that it can converge to the MMSE detector. We refer to such a modified version of our proposed filter as $\mathbf{G}_{\mathbf{p} \mu}{ }^{(m)}$ filter, where we avoid the generation of new interference and noise terms as in $\mathbf{G}_{\mathbf{p}}{ }^{(m)}$, while using the step sizes obtained for $\mathbf{G}_{\mu}{ }^{(m)}$ in [3]. Accordingly, we 
propose the modified version of the MMSE converging filter as

$$
\mathbf{y}_{\mathbf{p} \mu}^{(m)}=\underbrace{\left(\mu_{m} \mathbf{I}+\sum_{i=1}^{m-1} \mu_{m-i} \mathbf{J}_{i}\right)}_{\mathbf{G}_{\mathbf{p} \mu}(m)} \mathbf{y}^{(1)},
$$

where $\mathbf{J}_{i}$ is given by

$$
\mathbf{J}_{i}=\left[\mathbf{J}_{i-1}\left(\mathbf{I}-\mu_{K-i+1}\left(\mathbf{R}+\sigma^{2} \mathbf{I}\right)\right)\right]^{\odot}, \text { and } \mathbf{J}_{0}=\mathbf{I} \text {. }
$$

\section{E. A Weighted Matrix Filter, $\mathbf{G}_{\mathbf{p w}}{ }^{(m)}$}

In the $\mathbf{G}_{\mu}{ }^{(m)}$ and $\mathbf{G}_{\mathbf{p} \mu}{ }^{(m)}$ filters above, different step sizes are used in different stages (but the same step size for all users in a stage). Improved performance can be achieved if different scaling factors (weights) are used for different users in different stages. Accordingly, we propose a weighted version of our proposed filter $\mathbf{G}_{\mathbf{p}}{ }^{(m)}$. We refer to this weighted version as $\mathbf{G}_{\mathbf{p w}}{ }^{(m)}$, and is derived as follows.

In a weighted LPIC (WLPIC), the MAI estimate in a given stage is scaled by a weight before cancellation (unit weight corresponds to CLPIC and zero weight corresponds to MF). For example, the $m$ th stage output of the desired user $k, y_{k, \mathbf{w}}^{(m)}$, in a WLPIC is given by

$$
y_{k, \mathbf{w}}^{(m)}=y_{k}^{(1)}-w_{k}^{(m)} \sum_{j=1, j \neq k}^{K} \rho_{j k} y_{j, \mathbf{w}}^{(m-1)},
$$

where $w_{k}^{(m)}$ is the weight with which the MAI estimate for the $k$ th user in the $m$ th stage is scaled. For $m \geq 2$, the weighted cancellation operation in (31) can be written in the form

$$
\begin{aligned}
& \mathbf{y}_{\mathbf{w}}^{(m)}=\left(\mathbf{I}+\mathbf{W}^{(m)}(\mathbf{I}-\mathbf{R})\right. \\
&+\mathbf{W}^{(m)}(\mathbf{I}-\mathbf{R}) \mathbf{W}^{(m-1)}(\mathbf{I}-\mathbf{R})+\cdots \\
&\left.+\mathbf{W}^{(m)}(\mathbf{I}-\mathbf{R}) \mathbf{W}^{(m-1)}(\mathbf{I}-\mathbf{R}) \cdots \mathbf{W}^{(2)}(\mathbf{I}-\mathbf{R})\right) \mathbf{y}^{(1)},(32)
\end{aligned}
$$

where $\mathbf{W}^{(m)}$ is the weight matrix at the $m$ th stage, given by $\mathbf{W}^{(m)}=\operatorname{diag}\left(w_{1}^{(m)}, w_{2}^{(m)}, \cdots, w_{K}^{(m)}\right)$, and $\mathbf{W}^{(1)}=\mathbf{0}$. Now, as in $\mathbf{G}^{(m)}$, in order to avoid the generation of new interference and noise terms, we modify (32) as follows:

$$
\mathbf{y}_{\mathbf{p w}}^{(m)}=\underbrace{\left(\sum_{j=0}^{m-1} \tilde{\mathbf{B}}_{j}\right)}_{\mathbf{G}_{\mathbf{p w}}(m)} \mathbf{y}^{(1)},
$$

where

$$
\tilde{\mathbf{B}}_{n}=\left[\tilde{\mathbf{B}}_{n-1} \mathbf{W}^{(m-n+1)}(\mathbf{I}-\mathbf{R})\right]^{\odot} \text {, and } \tilde{\mathbf{B}}_{0}=\mathbf{I} .
$$

Note that $\mathbf{G}_{\mathbf{p w}}{ }^{(m)}$ becomes $\mathbf{G}_{\mathbf{p}}{ }^{(m)}$ when $\mathbf{W}^{(m)}=\mathbf{I}, \forall m>1$. Optimum Weight Matrix, $\mathbf{W}_{\text {opt }}^{(m)}$ : The $m$ th stage output of the $k$ th user when $\mathbf{G}_{\mathbf{p w}}{ }^{(m)}$ filter is used can be written as

$$
\begin{aligned}
& \left(y_{k}^{(m)}\right)_{\mathbf{G}_{\mathbf{p w}}}=y_{k}^{(1)}-w_{k}^{(m)} \sum_{i \neq k}^{K} q_{k, i}^{(m)} y_{i}^{(1)} \\
& =\underbrace{x_{k}\left(1-w_{k}^{(m)} \sum_{i \neq k}^{K} q_{k, i}^{(m)} \rho_{k i}\right)}_{\text {desired signal }}+\underbrace{n_{k}-w_{k}^{(m)} \sum_{i \neq k}^{K} q_{k, i}^{(m)} n_{i}}_{\text {noise }} \\
& +\underbrace{\sum_{i \neq k}^{K}\left(\rho_{k i}-w_{k}^{(m)}\left(q_{k, i}^{(m)}+\sum_{k_{1} \neq i, k}^{K} q_{k, k_{1}}^{(m)} \rho_{k_{1} i}\right)\right) x_{i}}_{\text {interference }},
\end{aligned}
$$

where

$$
\begin{aligned}
& q_{k, i}^{(m)}=\rho_{k i}-\sum_{k_{1} \neq k, i}^{K} w_{k_{1}}^{(m-1)} \rho_{k k_{1}} \rho_{k_{1} i} \\
& +\sum_{k_{1} \neq k, i}^{K} w_{k_{1}}^{(m-2)} \sum_{k_{2} \neq k, k_{1}}^{K} w_{k_{2}}^{(m-1)} \rho_{k k_{2}} \rho_{k_{2} k_{1}} \rho_{k_{1} i}-\cdots \\
& +(-1)^{m} \sum_{k_{1} \neq k, i}^{K} w_{k_{1}}^{(2)} \sum_{k_{2} \neq k, k_{1}}^{K} w_{k_{2}}^{(3)} \ldots \sum_{k_{m-2} \neq k, k_{m-3}}^{K} w_{k_{m-2}}^{(m-1)} \\
& \cdot \rho_{k k_{m-2}} \rho_{k_{m-2} k_{m-3}} \cdots \rho_{k_{3} k_{2}} \rho_{k_{2} k_{1}} \rho_{k_{1} i} .
\end{aligned}
$$

Since the interference and noise terms in on the RHS of (35) are the sum of linear combinations of complex Gaussian r.v's (since the fade coefficients $h_{k}$ are assumed to be complex Gaussian), the average SINR for the $k$ th user at the $m$ th stage output can be obtained, in closed-form, as

$$
\overline{\operatorname{SINR}}_{k}^{(m)}=\frac{A_{k}^{2}\left(1-a w_{k}^{(m)}\right)^{2}}{\sigma_{I}^{2}+\sigma_{N}^{2}},
$$

where

$$
\begin{aligned}
\sigma_{I}^{2} & =b+\left(w_{k}^{(m)}\right)^{2} c-2 w_{k}^{(m)} d, \\
\sigma_{N}^{2} & =\sigma^{2}\left(1+\left(w_{k}^{(m)}\right)^{2} e-2 w_{k}^{(m)} a\right), \\
a & =\sum_{i \neq k}^{K} q_{k, i}^{(m)} \rho_{k i}, \quad b=\sum_{i \neq k}^{K} \rho_{k i}^{2} A_{i}^{2}, \\
c & =\sum_{i \neq k}^{K}\left(q_{k, i}^{(m)}+\sum_{k_{1} \neq i, k}^{K} q_{k, k_{1}}^{(m)} \rho_{k_{1} i}\right)^{2} A_{i}^{2}, \\
d & =\sum_{i \neq k}^{K} \rho_{k i}\left(q_{k, i}^{(m)}+\sum_{k_{1} \neq i, k}^{K} q_{k, k_{1}}^{(m)} \rho_{k_{1} i}\right) A_{i}^{2}, \\
e & =\sum_{i \neq k}^{K} \sum_{j \neq k}^{K} q_{k, i}^{(m)} q_{k, j}^{(m)} \rho_{i j} .
\end{aligned}
$$

By differentiating the average SINR expression in (37) w.r.t. $w_{k}^{(m)}$ and equating to zero, the optimum weights $w_{k, o p t}^{(m)}$ can be obtained, in closed-form, as

$$
w_{k, o p t}^{(m)}=\frac{d-a b}{c-a d+\sigma^{2}\left(e-a^{2}\right)} .
$$

In Fig. 1, we plot the average SINR at the $m$ th stage output of the proposed $\mathbf{G}_{\mathbf{p w}}{ }^{(m)}$ filter, as a function of weight, $w_{k}^{(m)}$, at an average SNR of $20 \mathrm{~dB}$. The average SNR of user $k$ is 


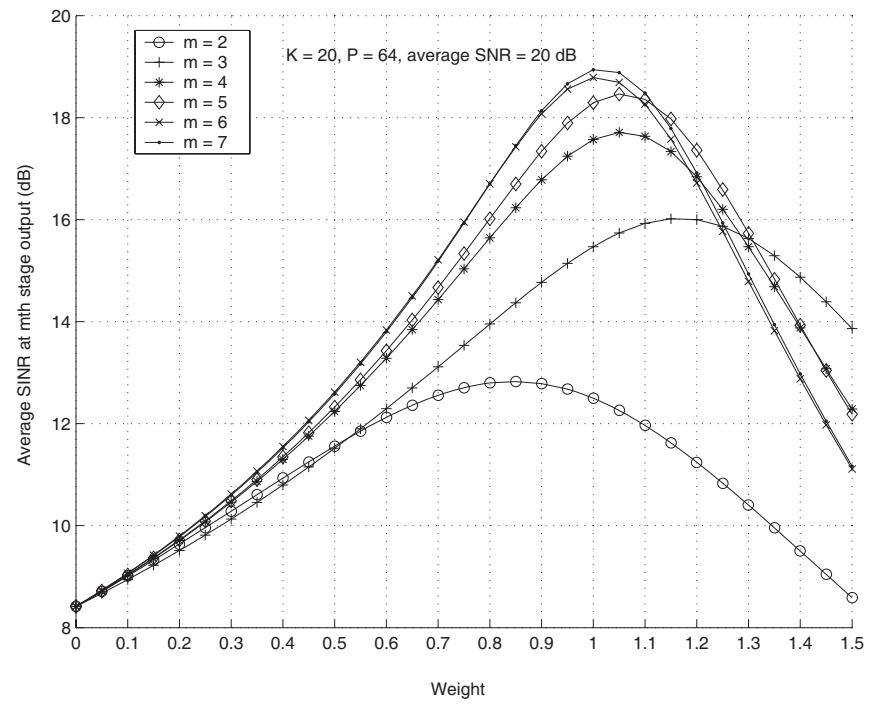

Fig. 1. Average output SINR as a function of weight $w_{k}^{(m)}$ for the proposed $\mathbf{G}_{\mathbf{p w}}{ }^{(m)}$ filter. $M=1$ (i.e., single carrier DS-CDMA), $K=20, P=64$, average SNR $=20 \mathrm{~dB}$. No near-far condition.

defined as $A_{k}^{2} / \sigma^{2}$. The number of users considered is $K=20$, the processing gain is $P=64$, and there is no near-far effect (i.e., $A_{1}=A_{2}=\cdots=A_{K}$ ). From Fig. 1 , it can be seen that for a given stage index $m$, the maximum output average SINR occurs at an optimum weight; the closed-form expression for this optimum weight is given by (38). The maximum average SINR increases as $m$ is increased. Also, we see diminishing improvement in SINR with increasing $m$, as expected. Another key observation in Fig. 1 is that, while non-unity weights are optimum for small values of $m$, the optimum weights approach unity for large $m$. $\mathbf{G}_{\mathbf{p w}}$ being structurally similar to $\mathbf{G}_{\mathbf{p}}$ except for the weights, like $\mathbf{G}_{\mathbf{p}}$ and $\mathbf{G}$ filters, $\mathbf{G}_{\mathbf{p w}}$ is also expected to converge to $\mathbf{R}^{-1}$ for $m \rightarrow \infty$, and this explains why $w_{k, o p t}^{(m)} \rightarrow$ 1 for $m \rightarrow \infty$.

\section{F. Results and Discussion}

In this subsection, we present a comparison of the bit error rate (BER) performance of different matrix filters. The various matrix filters considered include: $i$ ) the conventional filter, $\mathbf{G}^{(m)}$, given by (8), $\left.i i\right)$ the proposed filter, $\mathbf{G}_{\mathbf{p}}{ }^{(m)}$, given by (12), iii) the MMSE converging filter in [3], $\mathbf{G}_{\mu}{ }^{(m)}$, given by $(26), i v)$ the modified MMSE converging filter, $\mathbf{G}_{\mathbf{p} \mu}{ }^{(m)}$, given by (29), and $v$ ) the proposed weighted filter, $\mathbf{G}_{\mathbf{p w}}(m)$, given by (33).

In Fig. 2, we plot the BER performance of the conventional filter, $\mathbf{G}^{(m)}$, and the proposed filter, $\mathbf{G}_{\mathbf{p}}{ }^{(m)}$, as a function of the stage index, $m$, for $M=1, K=20, P=64$, and average $\mathrm{SNR}=15 \mathrm{~dB}$, for both no near-far (i.e., $A_{1}=A_{2}=\cdots=$ $\left.A_{K}\right)$ as well as near-far conditions. In all the simulations, user 1 is taken to be the desired user. Random binary sequences are used as spreading sequences. For the near-far condition, odd-indexed users (users $3,5,7, \cdots$ ) transmit with the same amplitude as the desired user 1 , whereas the even-indexed users (users $2,4,6, \cdots$ ) transmit at ten times larger amplitude than the desired user. The performance of the MF detector and the DC detector are also plotted for comparison. From Fig. 2, it can be seen that the conventional $\mathbf{G}^{(m)}$ filter approaches

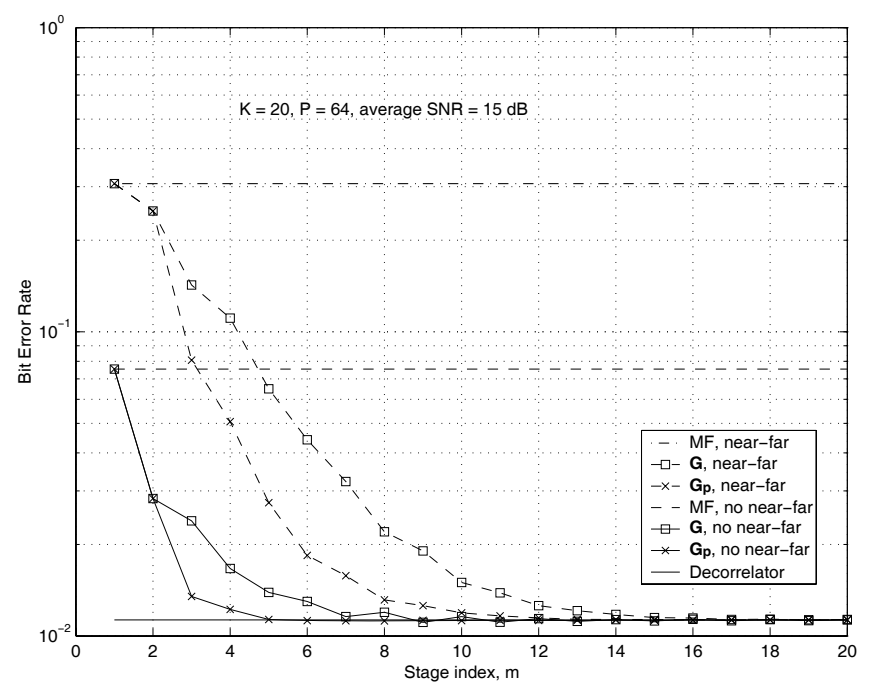

Fig. 2. BER performance of various linear matrix filters $-i$ ) conventional filter $\mathbf{G}^{(m)}$, and $\left.i i\right)$ proposed filter $\mathbf{G}_{\mathbf{p}}{ }^{(m)} \cdot M=1, K=20, P=64$, average $\mathrm{SNR}=15 \mathrm{~dB}$. Near-far as well as no near-far conditions.

the DC detector performance rather slowly for increasing $m$. Observe that the performance of the proposed $\mathbf{G}_{\mathbf{p}}{ }^{(m)}$ filter and the conventional $\mathbf{G}^{(m)}$ filter are the same for $m=2$ because of no desired signal loss recovery at the 2 nd stage of both $\mathbf{G}^{(m)}$ and $\mathbf{G}_{\mathbf{p}}{ }^{(m)}$. However, for $m \geq 3$, the $\mathbf{G}_{\mathbf{p}}{ }^{(m)}$ filter performs better than the $\mathbf{G}^{(m)}$ filter. This is because the $\mathbf{G}_{\mathbf{p}}{ }^{(m)}$ filter, as intended, avoids the generation of new interference and noise terms (e.g., $B_{I}$ and $B_{N}$ terms for $m=3$ ) compared to the $\mathbf{G}^{(m)}$ filter. The $\mathbf{G}_{\mathbf{p}}{ }^{(m)}$ filter is found to offer greater advantage in near-far conditions, since strong other-user interference terms in $B_{I}$ are avoided in the $\mathbf{G}_{\mathbf{p}}^{(m)}$.

Next, in Fig. 3, we present a comparison of the performance of the MMSE converging $\mathbf{G}_{\mu}{ }^{(m)}$ filter in [3], and the modified MMSE converging, $\mathbf{G}_{\mathbf{p} \mu}{ }^{(m)}$, for the same system conditions in Fig. 2. The performance of the MF and MMSE detectors are also plotted for comparison. Here again, the $\mathbf{G}_{\mu}{ }^{(m)}$ and $\mathbf{G}_{\mathbf{p} \mu}{ }^{(m)}$ filters perform the same for $m=2$. Also, both $\mathbf{G}_{\mu}{ }^{(m)}$ and $\mathbf{G}_{\mathbf{p} \mu}{ }^{(m)}$ are seen to approach the MMSE performance as $m$ is increased. For $m \geq 3, \mathbf{G}_{\mathbf{p} \mu}{ }^{(m)}$ performs better than $\mathbf{G}_{\mu}{ }^{(m)}$ because of the avoidance of new interference and noise terms. In generating the plot for $\mathbf{G}_{\mathbf{p} \mu}{ }^{(m)}$, we have used the step sizes in (28), which are actually optimum for $\mathbf{G}_{\mu}{ }^{(m)}$. Even with these step sizes (which can be suboptimum for $\left.\mathbf{G}_{\mathbf{p} \mu}{ }^{(m)}\right)$, the proposed $\mathbf{G}_{\mathbf{p} \mu}{ }^{(m)}$ filter approaches the MMSE performance faster than the $\mathbf{G}_{\mu}{ }^{(m)}$ filter.

Finally, in Fig. 4, we illustrate the performance of all the matrix filters considered in this paper, including the proposed weighted filter, $\mathbf{G}_{\mathbf{p w}}{ }^{(m)}$, under the no near-far condition. The performance of the MF, DC and MMSE detectors are also plotted. It can be observed that among all the filters considered, the proposed weighted filter $\mathbf{G}_{\mathbf{p w}}{ }^{(m)}$ performs the best for small values of $m$ ( $m<6$, for example). In other words, $\mathbf{G}_{\mathbf{p w}}{ }^{(m)}$ performs best in terms of convergence, i.e., fewer stages are sufficient to yield close to DC detector performance. This may be expected, because in the $\mathbf{G}_{\mu}{ }^{(m)}$ and $\mathbf{G}_{\mathbf{p} \mu}{ }^{(m)}$ filters the optimum step sizes are obtained only on a 


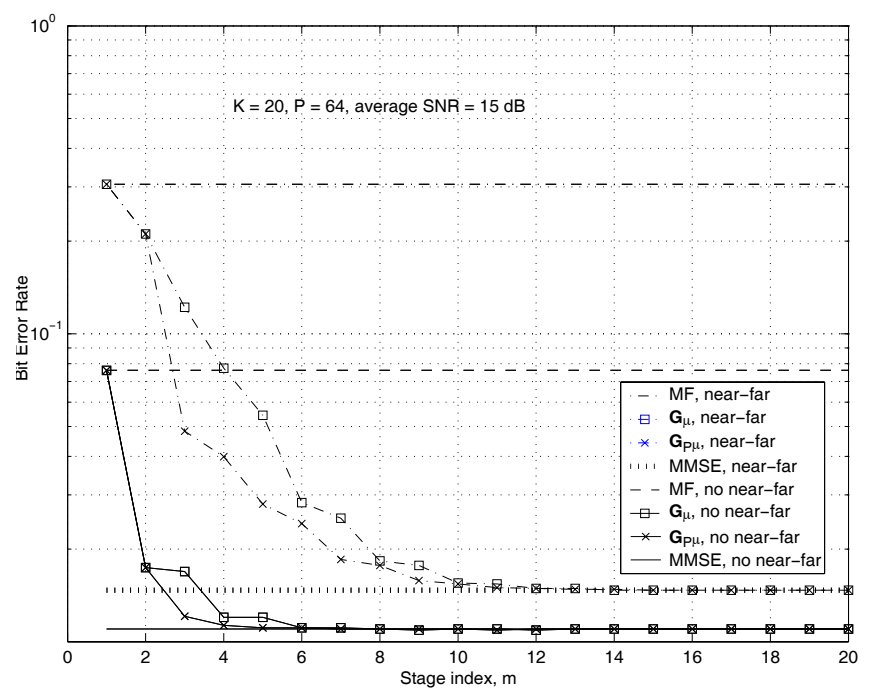

Fig. 3. BER performance of various linear matrix filters $-i$ ) MMSE converging filter $\mathbf{G}_{\mu}{ }^{(m)}$, and $\left.i i\right)$ modified MMSE converging filter, $\mathbf{G}_{\mathbf{p} \mu}{ }^{(m)}$. $M=1, K=20, P=64$, average SNR $=15 \mathrm{~dB}$. Near-far as well as no near-far conditions.

per-stage basis, whereas in the $\mathbf{G}_{\mathbf{p w}}{ }^{(m)}$ filter the optimum weights are obtained on a per-stage as well as a per-user basis. The computation of the optimum weights, $w_{k, o p t}^{(m)}$, for the $\mathbf{G}_{\mathbf{p w}}{ }^{(m)}$ filter, using the closed-form expression in (38), adds to the receiver complexity. However, since these optimum weights are computed by using the average SINR expression, the weights computation can be carried out off-line once (or whenever users exit from or enter into the system, which changes the correlation matrix), and this need not add to the per-bit complexity of the canceller. In Fig. 4, we also show the performance of the conventional weighted LPIC given in [9], denoted by $\mathbf{G}_{\mathbf{w}}{ }^{(m)}$, for up to $m=4$. As can be seen, because of the SIR maximization using optimum weights, the performance of the $\mathbf{G}_{\mathbf{W}}{ }^{(m)}$ filter is almost the same as that of the proposed $\mathbf{G}_{\mathbf{p w}}{ }^{(m)}$ filter. We further note that the optimum weights expressions for the $\mathbf{G}_{\mathbf{w}}{ }^{(m)}$ filter need to be derived separately on a stage by stage basis - the optimum weights expressions for up to $m=4$ are given in [9],[10], and the optimum weights derivation becomes increasingly cumbersome for increasing $m$. On the other hand, the feature of making the diagonal elements zero in the $\mathbf{G}_{\mathbf{p w}}{ }^{(m)}$ filter allows optimum weights expressions to be obtained for any $m$ (given by Eqn. (38)). In terms of convergence as well as complexity, the proposed filter $\mathbf{G}_{\mathbf{p} \mu}{ }^{(m)}$ is also quite attractive.

\section{Proposed Filters in Multicarrier DS-CDMA}

In this section, we extend the proposed matrix filter solutions in the previous section to multicarrier DS-CDMA (i.e., for $M \geq 2$ ). Here, the multicarrier combining (MCC) operation has to be performed in addition to LPIC. Depending on which operation (i.e., MCC or LPIC) gets done first at the receiver, the resulting performances and complexities differ.

Type-I Receiver: We first consider a receiver where we perform LPIC first on each subcarrier, followed by MCC as shown in Fig. 5 [12]. We refer to this receiver as TypeI receiver. We note that with this Type-I receiver using the

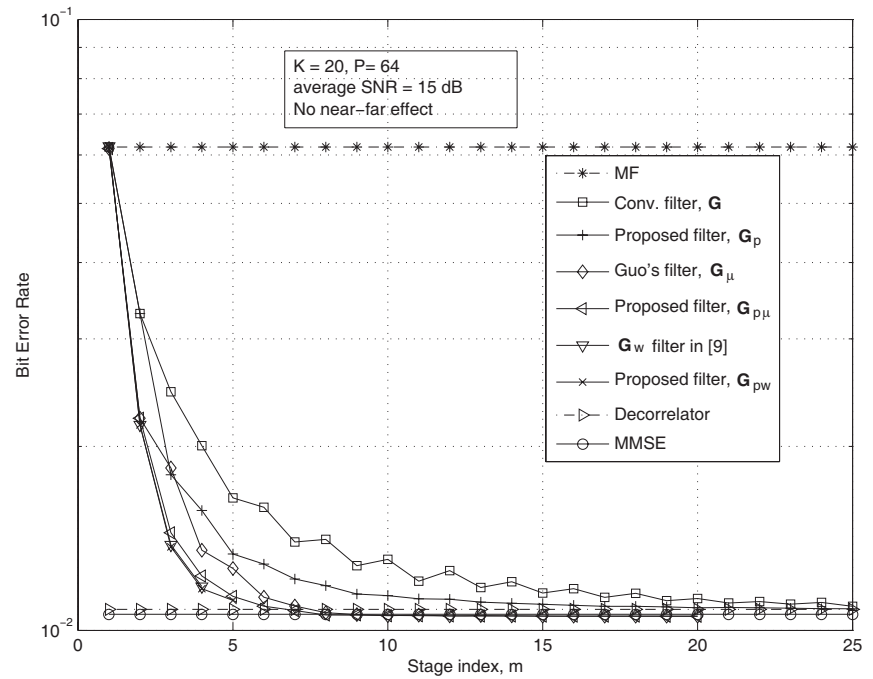

Fig. 4. BER performance of various linear matrix filters $-i) \mathbf{G}^{(m)}$ filter, $\left.i i\right)$ proposed $\mathbf{G}_{\mathbf{p}}{ }^{(m)}$ filter, iii) MMSE converging $\mathbf{G}_{\mu}{ }^{(m)}$ filter, $i v$ ) modified MMSE converging filter, $\left.\mathbf{G}_{\mathbf{p} \mu}{ }^{(m)}, v\right)$ proposed weighted filter, $\mathbf{G}_{\mathbf{p w}}{ }^{(m)}$, and $v i$ ) conventional weighted LPIC filter in [9], $\mathbf{G}_{\mathbf{w}}{ }^{(m)} \cdot M=1, K=20$, $P=64$, average $\mathrm{SNR}=15 \mathrm{~dB}$. No near-far condition.

conventional filter $\mathbf{G}^{(m)}$, tractable BER analysis becomes feasible (reported in [12]). Also, this receiver architecture can be viewed as a direct adoption of the filters proposed for single carrier DS-CDMA in Sec. III, on individual subcarriers in the MC DS-CDMA system. Hence, all the matrix filters in Sec. III, namely, $\mathbf{G}_{\mathbf{p}}{ }^{(m)}, \mathbf{G}_{\mu}^{(m)}, \mathbf{G}_{\mathbf{p} \mu}{ }^{(m)}$ and $\mathbf{G}_{\mathbf{p w}}{ }^{(m)}$, can be directly employed on the individual subcarriers.

Type-II Receiver: Since MCC operation can provide frequency diversity, performing MCC before LPIC can enhance the accuracy of the estimates of the MAI and hence improve performance. Accordingly, we propose a Type-II receiver, where MCC is performed first, followed by LPIC, as shown in Fig. 6. The output of the MC combiner in vector form, denoted by $\mathbf{y}^{c(1)}$, can be written as

$$
\mathbf{y}^{c(1)}=\mathbf{R}^{c} \mathbf{b}+\mathbf{z}
$$

where $\mathbf{y}^{c(1)}=\left[\begin{array}{llll}y_{1}^{c(1)} & y_{2}^{c(1)} & \ldots & y_{K}^{c(1)}\end{array}\right]^{T}, \quad y_{k}^{c(1)}=$ $\sum_{i=1}^{M}\left(h_{k}^{(i)}\right)^{*} y_{k}^{(1),(i)}, \mathbf{R}^{c} \triangleq \sum_{i=1}^{M}\left(\mathbf{H}^{(i)}\right)^{H} \mathbf{R}^{(i)} \mathbf{H}^{(i)}$, and $\mathbf{z}=$ $\left[\sum_{i=1}^{M}\left(h_{1}{ }^{(i)}\right)^{*} n_{1}^{(i)} \sum_{i=1}^{M}\left(h_{2}{ }^{(i)}\right)^{*} n_{2}^{(i)} \cdots \sum_{i=1}^{M}\left(h_{K}{ }^{(i)}\right)^{*} n_{K}^{(i)}\right]^{T}$. The conventional filter at the MCC output (referred to as $\mathbf{G}^{c(m)}$ filter) has its $m$ th stage output vector given by

$$
=\underbrace{\left(\mathbf{I}+\sum_{i=1}^{m-1}\left(\mathbf{I}-\mathbf{R}_{\mathbf{e f f}}\right)^{i}\right)}_{\mathbf{G}^{c(m)}} \mathbf{y}^{c(1)},
$$

where $\mathbf{R}_{\text {eff }} \triangleq \mathbf{R}^{c} \mathbf{H}_{\mathbf{D}}{ }^{-1}$, and $\mathbf{H}_{\mathbf{D}}=\sum_{i=1}^{M}\left(\mathbf{H}^{(i)}\right)^{H} \mathbf{H}^{(i)}$. Similar to the proposed $\mathbf{G}_{\mathbf{p}}{ }^{(m)}$ filter for single carrier DSCDMA in the previous section (where the idea of zeroing the diagonal elements of a certain matrix is adopted), a proposed matrix filter for MC DS-CDMA Type-II receiver, denoted by 


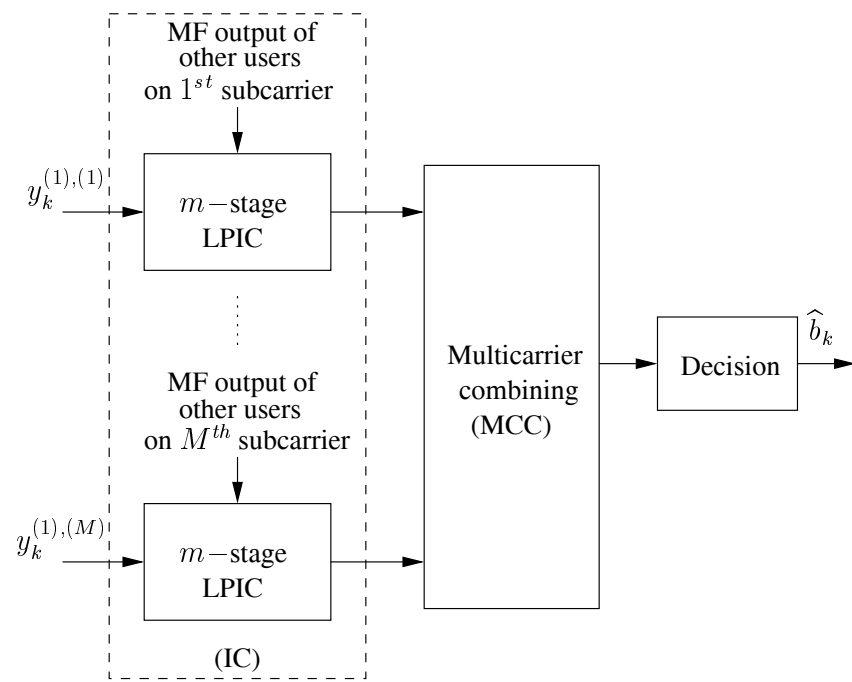

Fig. 5. Type-I Receiver for multicarrier DS-CDMA, $M \geq 2$. LPIC is done first on each subcarrier. Multicarrier Combining is done next.

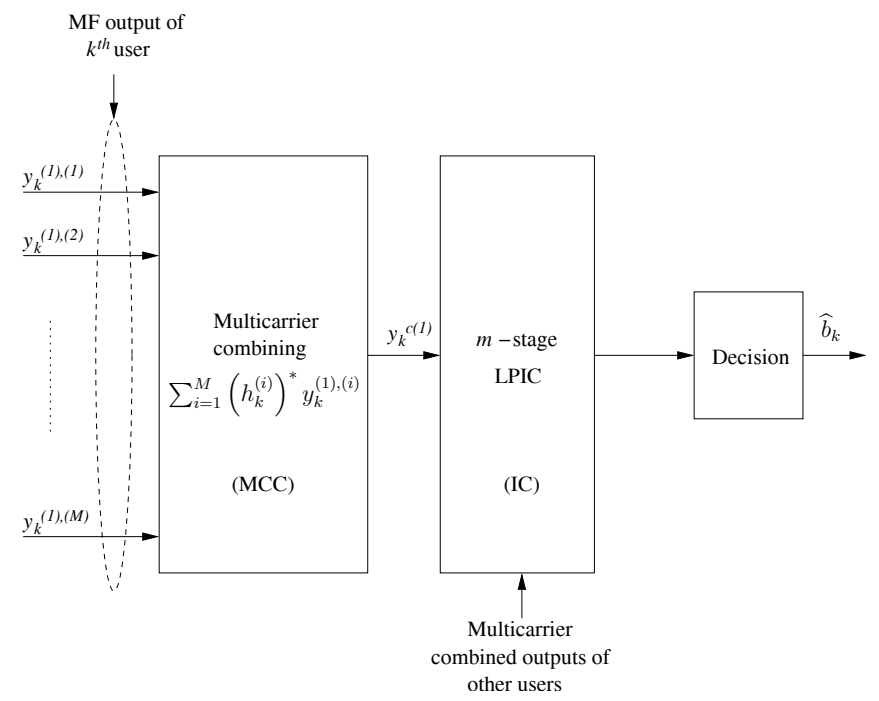

Fig. 6. Type-II Receiver for MC DS-CDMA, $M \geq 2$. LPIC is done after multicarrier combining.

$\mathbf{G}_{\mathbf{p}}{ }^{c(m)}$, can be obtained as

$$
\mathbf{y}_{\mathbf{p}}^{c(m)}=\underbrace{\left(\sum_{j=0}^{m-1} \mathbf{B}_{j}^{c}\right)}_{\mathbf{G}_{\mathbf{p}}{ }^{c(m)}} \mathbf{y}^{c(1)},
$$

where $\mathbf{B}_{n}^{c}=\left[\mathbf{B}_{n-1}^{c}\left(\mathbf{I}-\mathbf{R}_{\mathrm{eff}}\right)\right]^{\odot}$, and $\mathbf{B}_{0}^{c}=\mathbf{I}$.

\section{A. Results and Discussions}

In Fig. 7, we present the simulated BER performance comparison between the Type-I and Type-II receivers for MC DS-CDMA using different matrix filters. Specifically, we compare the performance of $i$ ) Type-I receiver with the $\mathbf{G}^{(m)}$ filter, $i i)$ Type-I receiver with the $\mathbf{G}_{\mathbf{p}}{ }^{(m)}$ filter, $\left.i i i\right)$ Type-II receiver with the $\mathbf{G}^{c(m)}$ filter, and $\left.i v\right)$ Type-II receiver with the $\mathbf{G}_{\mathbf{p}}{ }^{c(m)}$ filter. We also compare the performance of the above detectors with DC and MMSE detectors. Random binary sequences of length $P$ are used as the spreading sequences

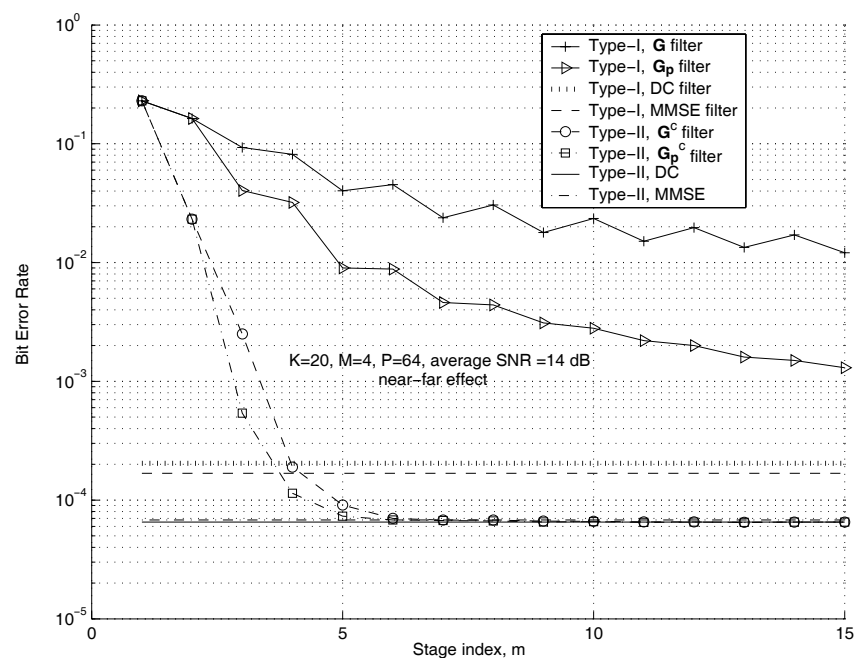

Fig. 7. BER performance of the proposed Type-I and Type-II receivers as a function of stage index $m$ for MC DS-CDMA with $-i$ ) Type-I with $\mathbf{G}^{(m)}$ filter, $i i)$ Type-I with $\mathbf{G}_{\mathbf{p}}{ }^{(m)}$ filter, $\left.i i i\right)$ Type-II with $\mathbf{G}^{c(m)}$ filter, and $i v$ ) Type-II with $\mathbf{G}_{\mathbf{p}}{ }^{c(m)}$ filter. $M=4, K=20, P=64$, average $\mathrm{SNR}=14$ dB. Near-far effect as described in Sec. III-F.

on each subcarrier, and the average SNR of user $k$ is defined as $\frac{M A_{k}^{2}}{\sigma^{2}}$. In Fig. 7, we plot the BER as a function of stage index, $m$, for $M=4, K=20, P=64$, and average SNR = $14 \mathrm{~dB}$. The following observations can be made from Fig. 7:

- Comparing the performance of Type-I and Type-II receivers for a given filter, we observe that Type-II receivers perform significantly better than Type-I receivers. For example, comparing the performance of Type-I receiver with the $\mathbf{G}^{(m)}$ filter and Type-II receiver with the $\mathbf{G}^{c(m)}$ filter, we see that Type-II receiver with the $\mathbf{G}^{c(m)}$ filter performs significantly better (e.g., for $m=4$, Type-I with the $\mathbf{G}^{(m)}$ filter gives a BER of $8 \times 10^{-2}$, whereas TypeII with the $\mathbf{G}^{c(m)}$ filter results in a BER of $\left.2 \times 10^{-4}\right)$. The superiority of Type-II receivers is consistent across all filters considered, i.e., $\mathbf{G}, \mathbf{G}_{\mathrm{p}}$, DC, MMSE. This superiority of Type-II receivers is expected, since the MAI estimates can be more accurate in Type-II, because of frequency diversity effect of multicarrier combining before IC.

- Like in SC DS-CDMA, in MC DS-CDMA, the proposed $\mathbf{G}_{\mathbf{p}}$ filter performs better than the $\mathbf{G}$ filter. This is observed to be true in both Type-I as well as TypeII receivers. For example, Type-I with the $\mathbf{G}_{\mathbf{p}}{ }^{(m)}$ filter achieves a BER of $9 \times 10^{-3}$ in just 5 stages, whereas the same BER is achieved by Type-I with the $\mathbf{G}^{(m)}$ filter only after 15 stages. Likewise, in Type-II receivers, the $\mathbf{G}_{\mathbf{p}}{ }^{c(m)}$ filter performs better than the $\mathbf{G}^{c(m)}$ filter.

\section{Conclusions}

We proposed improved LPICs for CDMA by viewing an LPIC as a linear matrix filter. Specifically, we proposed new linear matrix filters which achieved better performance than other linear matrix filters in the literature. This was made possible by avoiding the generation of certain new interference and noise terms by making the diagonal elements of a certain matrix equal to zero in each stage, without adding complexity. 
We also extended the proposed matrix filter solutions to multicarrier DS-CDMA, where we considered two types of receivers; in both types of receivers the proposed filters were shown to outperform other filters in the literature.

\section{APPENDIX A}

\section{EXPRESSION FOR THE 3 RD STAGE $\mathbf{G}^{(m)}$ FILTER OUTPUT}

In this appendix, we write the expression for the 3rd stage output of the $\mathbf{G}^{(m)}$ filter (i.e., CLPIC) in an expanded form. From (8), $\mathbf{y}^{(3)}$ can be written as

$$
\begin{aligned}
\mathbf{y}^{(3)} & =\left[\mathbf{I}+(\mathbf{I}-\mathbf{R})+(\mathbf{I}-\mathbf{R})^{2}\right] \mathbf{y}^{(1)} \\
& =\mathbf{y}^{(2)}+(\mathbf{I}-\mathbf{R})^{2} \mathbf{y}^{(1)} .
\end{aligned}
$$

Equivalently,

$$
\begin{aligned}
y_{k}^{(3)} & =y_{k}^{(2)}+\sum_{j=1}^{K} \sum_{i \neq k, j}^{K} \rho_{k i} \rho_{i j} y_{j}^{(1)} \\
& =y_{k}^{(2)}+\underbrace{\sum_{i \neq k}^{K} \rho_{k i} \rho_{i k} y_{k}^{(1)}}_{T_{1} \text { : case of } j=k}+\underbrace{\sum_{j \neq k}^{K} \sum_{i \neq k, j}^{K} \rho_{k i} \rho_{i j} y_{j}^{(1)}}_{T_{2} \text { : case of } j \neq k} .
\end{aligned}
$$

We point out that the term $T_{1}$ in the above equation recovers the desired signal lost in the 2nd stage, and the term $T_{2}$ removes the interference terms generated in the 2 nd stage. Substituting (9) and (5) in (43), we can write $y_{k}^{(3)}$ as shown in (44) given at the bottom of this page.

\section{APPENDIX B}

EXPRESSION FOR $m$ TH STAGE $\mathbf{G}^{(m)}$ FILTER OUTPUT, $m>3$

From (8), we can write $y_{k}^{(m)}$ for $m \geq 3$ as

$$
\begin{aligned}
y_{k}^{(m)}= & y_{k}^{(1)}-\left(\sum_{k_{1} \neq k}^{K} \rho_{k k_{1}}-\sum_{k_{1}=1}^{K}\left(\sum_{k_{2} \neq k, k_{1}}^{K} \rho_{k k_{2}} \rho_{k_{2} k_{1}}\right.\right. \\
& -\sum_{k_{2} \neq k_{1}}^{K} \sum_{k_{3} \neq k, k_{2}}^{K} \rho_{k k_{3}} \rho_{k_{3} k_{2}} \rho_{k_{2} k_{1}}+\cdots \\
& +(-1)^{m-1} \sum_{k_{2} \neq k_{1}}^{K} \sum_{k_{3} \neq k_{2}}^{K} \cdots \sum_{k_{m-1} \neq k, k_{m-2}}^{K} \\
& \left.\left.\rho_{k k_{m-1}} \rho_{k_{m-1} k_{m-2}} \cdots \rho_{k_{3} k_{2}} \rho_{k_{2} k_{1}}\right)\right) y_{k_{1}}^{(1)} .
\end{aligned}
$$

Equivalently, (45) can be written as

$$
\begin{aligned}
y_{k}^{(m)}= & y_{k}^{(m-1)}+(-1)^{m+1} \sum_{k_{1}=1}^{K} \sum_{k_{2} \neq k_{1}}^{K} \sum_{k_{3} \neq k_{2}}^{K} \cdots \sum_{k_{m-2} \neq k_{m-3}}^{K} \\
& \sum_{k_{m-1} \neq k, k_{m-2}}^{K} \rho_{k k_{m-1}} \rho_{k_{m-1}} k_{m-2} \cdots \rho_{k_{3} k_{2}} \rho_{k_{2} k_{1}} y_{k_{1}}^{(1)} .
\end{aligned}
$$

The first summation in the 2nd term on the RHS of (46) can be split into two terms, one for $k_{1}=k$ and another for $k_{1} \neq k$, as shown in (47) given at the end of the next page. For $m \geq 4$, $y_{k}^{(m-1)}$ can be written in an alternate form as shown in (48) given at the end of the next page. Comparing the output terms in stages $m-1$ and $m$ in Eqns. (48) and (47), respectively, we can observe the following.

- The desired signal loss/gain that occurred in the $(m-1)$ th stage (i.e., $T_{5}$ in (48)) is recovered/removed in the $m$ th stage (see $T_{3}$ in (47) and note that $y_{k}^{(1)}$ in it has $x_{k}$ ). In the process, new interference terms proportional to $\rho^{m}$ and additional noise terms proportional to $\rho^{m-1}$ get generated, due to all terms other than $x_{k}$ in $y_{k}^{(1)}$ in $T_{3}$. Note that for the case of $m=3$, these interference

$$
\begin{aligned}
& y_{k}^{(3)}=x_{k}\left(1-\sum_{j \neq k}^{K} \rho_{k j} \rho_{j k}\right)-\sum_{j \neq k}^{K} \sum_{l \neq j, k}^{K} \rho_{k j} \rho_{j l} x_{l}+n_{k}-\sum_{j \neq k}^{K} \rho_{k j} n_{j}+\sum_{i \neq k}^{K} \rho_{k i} \rho_{i k}\left[x_{k}+\sum_{j \neq k}^{K} \rho_{k j} x_{j}+n_{k}\right] \\
& +\sum_{j \neq k}^{K} \sum_{i \neq k, j}^{K} \rho_{k i} \rho_{i j}\left[x_{j}+\sum_{l \neq j}^{K} \rho_{j l} x_{l}+n_{j}\right] \\
& =x_{k}-\sum_{j \neq k}^{K} \rho_{k j} \rho_{j k} x_{k}-\sum_{j \neq k}^{K} \sum_{l \neq j, k}^{K} \rho_{k j} \rho_{j l} x_{l}+n_{k}-\sum_{j \neq k}^{K} \rho_{k j} n_{j}+\sum_{i \neq k}^{K} \rho_{k i} \rho_{i k} x_{k}+\sum_{i \neq k}^{K} \rho_{k i} \rho_{i k} \sum_{j \neq k}^{K} \rho_{k j} x_{j}+\sum_{i \neq k}^{K} \rho_{k i} \rho_{i k} n_{k} \\
& +\sum_{j \neq k}^{K} \sum_{i \neq k, j}^{K} \rho_{k i} \rho_{i j} x_{j}+\sum_{j \neq k}^{K} \sum_{i \neq k, j}^{K} \rho_{k i} \rho_{i j} \sum_{l \neq j}^{K} \rho_{j l} x_{l}+\sum_{j \neq k}^{K} \sum_{i \neq k, j}^{K} \rho_{k i} \rho_{i j} n_{j} \\
& =x_{k}-\underbrace{\sum_{j \neq k}^{K} \rho_{k j} \rho_{j k} x_{k}}_{\mathrm{A}}-\underbrace{\sum_{j \neq k}^{K} \sum_{l \neq j, k}^{K} \rho_{k j} \rho_{j l} x_{l}}_{\mathrm{C}}+n_{k}-\sum_{j \neq k}^{K} \rho_{k j} n_{j}+\underbrace{\sum_{i \neq k}^{K} \rho_{k i} \rho_{i k} x_{k}}_{\mathrm{A}}+\underbrace{\sum_{i \neq k}^{K} \rho_{k i} \rho_{i k} \sum_{j \neq k}^{K} \rho_{k j} x_{j}}_{B_{I}}+\underbrace{\sum_{i \neq k}^{K} \rho_{k i} \rho_{i k} n_{k}}_{B_{N}} \\
& +\underbrace{\sum_{j \neq k}^{K} \sum_{i \neq k, j}^{K} \rho_{k i} \rho_{i j} x_{j}}_{\text {व }}+\underbrace{\sum_{j \neq k}^{K} \sum_{i \neq k, j}^{K} \rho_{k i} \rho_{i j} \rho_{j k} x_{k}}_{\text {D }}+\underbrace{\sum_{j \neq k}^{K} \sum_{i \neq k, j}^{K} \rho_{k i} \rho_{i j} \sum_{l \neq k, j}^{K} \rho_{j l} x_{l}}_{E_{I}}+\underbrace{\sum_{j \neq k}^{K} \sum_{i \neq k, j}^{K} \rho_{k i} \rho_{i j} n_{j}}_{E_{N}} .
\end{aligned}
$$




$$
\begin{aligned}
& y_{k}^{(m)}=y_{k}^{(m-1)}+\underbrace{(-1)^{m+1} \sum_{k_{2} \neq k}^{K} \sum_{k_{3} \neq k_{2}}^{K} \cdots \sum_{k_{m-2} \neq k_{m-3}}^{K} \sum_{k_{m-1} \neq k, k_{m-2}}^{K} \rho_{k k_{m-1}} \rho_{k_{m-1} k_{m-2}} \cdots \rho_{k_{3} k_{2}} \rho_{k_{2} k} y_{k}^{(1)}}_{T_{3}} \\
& +\underbrace{(-1)^{m+1} \sum_{k_{1} \neq k}^{K} \sum_{k_{2} \neq k_{1}}^{K} \sum_{k_{3} \neq k_{2}}^{K} \cdots \sum_{k_{m-2} \neq k_{m-3}}^{K} \sum_{k_{m-1} \neq k, k_{m-2}}^{K} \rho_{k k_{m-1}} \rho_{k_{m-1} k_{m-2}} \cdots \rho_{k_{3} k_{2}} \rho_{k_{2} k_{1}} y_{k_{1}}^{(1)}}_{T_{4}} . \\
& y_{k}^{(m-1)}=x_{k}+\underbrace{(-1)^{m} \sum_{k_{2} \neq k}^{K} \sum_{k_{3} \neq k_{2}}^{K} \cdots \sum_{k_{m-2} \neq k_{m-3}}^{K} \sum_{k_{m-1} \neq k, k_{m-2}}^{K} \rho_{k k_{m-1}} \rho_{k_{m-1} k_{m-2}} \cdots \rho_{k_{3} k_{2}} \rho_{k_{2} k} x_{k}}_{T_{5}: \text { desired signal loss/gain at the (m-1)th stage output }} \\
& +\underbrace{(-1)^{m} \sum_{k_{1} \neq k}^{K} \sum_{k_{2} \neq k_{1}}^{K} \sum_{k_{3} \neq k_{2}}^{K} \cdots \sum_{k_{m-2} \neq k_{m-3}}^{K} \sum_{k_{m-1} \neq k, k_{m-2}}^{K} \rho_{k k_{m-1}} \rho_{k_{m-1} k_{m-2}} \cdots \rho_{k_{3} k_{2}} \rho_{k_{2} k_{1}} x_{k_{1}}} \\
& T_{6} \text { : new interference terms generated at the (m-1)th stage output } \\
& +n_{k}-\sum_{k_{1} \neq k}^{K} \rho_{k k_{1}} n_{k_{1}}+\sum_{k_{1}=1}^{K} \sum_{k_{2} \neq k, k_{1}} \rho_{k k_{2}} \rho_{k_{2} k_{1}} n_{k_{1}}-\cdots \\
& +(-1)^{m} \sum_{k_{1}=1}^{K} \sum_{k_{2} \neq k_{1}}^{K} \sum_{k_{3} \neq k_{2}}^{K} \cdots \sum_{k_{m-3} \neq k_{m-4}}^{K} \sum_{k_{m-2} \neq k, k_{m-3}}^{K} \rho_{k k_{m-2}} \rho_{k_{m-2} k_{m-3}} \cdots \rho_{k_{3} k_{2}} \rho_{k_{2} k_{1}} n_{k_{1}} \text {. }
\end{aligned}
$$

and noise terms generated are given by $B_{I}$ and $B_{N}$ in Eqn. (44) in Appendix A.

- The new interference terms that were generated in the $(m-1)$ th stage (i.e., $T_{6}$ in (48)) are removed in the $m$ th stage (see $T_{4}$ in (47) and note that $y_{k_{1}}^{(1)}$ in it has $x_{k_{1}}$ ). In the process, new interference terms proportional to $\rho^{m}$ and additional noise terms proportional to $\rho^{m-1}$ get generated, due to all terms other than $x_{k_{1}}$ in $y_{k_{1}}^{(1)}$ in $T_{4}$. Note that for the case of $m=3$, these interference and noise terms are given by $E_{I}$ and $E_{N}$ in Eqn. (44) in Appendix A.

\section{REFERENCES}

[1] D. Divsalar, M. K. Simon, and D. Raphaeli, "Improved parallel interference cancellation for CDMA," IEEE Trans. Commun., vol. 46, no. 2, pp. 258-268, Feb. 1998.

[2] D. Guo, L. K. Rasmussen, and T. J. Lim, "Linear parallel interference cancellation in long-code CDMA multiuser detection," IEEE J. Select. Areas Commun., vol. 17, no. 12, pp. 2074-2081, Dec. 1999.

[3] D. Guo, L. K. Rasmussen, S. Sun, and T. J. Lim, "A matrix algebraic approach to linear parallel interference cancellation in CDMA," IEEE Trans. Commun., vol. 48, no. 1, pp. 152-161, Jan. 2000.

[4] D. R. Brown, M. Motani, V. Veeravalli, H. V. Poor, and C. R. Johnson, Jr., "On the performance of linear parallel interference cancellation," IEEE Trans. Inform. Theory, vol. 47, no. 5, pp. 1957-1970, July 2001.

[5] C.-H. Hwang, C.-S. Kim, and C.-C. J. Kuo, "Analysis of multistage linear parallel interference cancellation in CDMA systems using graphical representation," in Proc. IEEE ICC'2002, Apr. 2002.

[6] G. F. Trichard, J. S. Evans, and I. B. Collings, "Large system analysis of linear multistage parallel interference cancellation," IEEE Trans. Commun., vol. 50, no. 11, pp. 1778-1786, Nov. 2002.

[7] D. Guo, S. Verdu, and L. K. Rasmussen, "Asymptotic normality of linear multiuser receiver outputs," IEEE Trans. Inform. Theory, vol. 48, no. 12, pp. 3080-3094, Dec. 2002.

[8] S. Moshavi, E. G. Kanterakis, and D. L. Schilling, "Multistage linear receivers for DS-CDMA systems," Intl. J. Wireless Inform. Networks, vol. 3, no. 1, pp. 1-17, 1996.
[9] V. Tikiya, S. Manohar, A. Chockalingam, "SIR-optimized weighted linear parallel interference canceller on fading channels," IEEE Trans. Wireless Commun., vol. 5, no. 8, pp. 1998-2003, Aug. 2006.

[10] S. Manohar, "Design and analysis of interference cancellers in CDMA and OFDMA systems," M. E. thesis, Department of ECE, Indian Institute of Science, Bangalore, June 2005.

[11] M. Ghotbi and M. R. Soleymani, "A simple method for computing partial cancellation factors in CDMA using PPIC receiver," in Proc. IEEE ICASSP, pp. 973-976, 2004.

[12] S. Manohar, V. Tikiya, R. Annavajjala, and A. Chockalingam, "BERoptimal linear parallel interference cancellation for multicarrier DSCDMA in Rayleigh fading," IEEE Trans. Commun., vol. 55, no. 6, pp. 1253-1265, June 2007.

[13] L. Hanzo, L.-L. Yang, E.-L. Kuan, and K. Yen, Single- and MultiCarrier DS-CDMA: Multiuser Detection, Space-Time Spreading, Synchronization and Standards. IEEE Press, 2003.

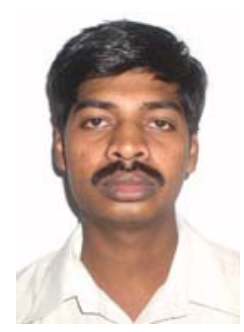

Thati Srikanth received his Master of Engineering degree in Electrical Communication Engineering from the Indian Institute of Science, Bangalore, India, in 2006, and the Bachelor of Technology degree in Electronics and Communication Engineering from the V. R. Siddhartha Engineering College, Vijayawada, Andhra Pradesh, India, in 2003. From July 2006 to April 2008, he worked with Honeywell Technology Solutions Lab Private Limited, Bangalore, India, as a senior engineer. $\mathrm{He}$ is currently working with ORCA Radio Systems Private Limited, Bangalore, India. His research interests include indoor channel modeling, WPAN, CDMA, and MIMO systems. 


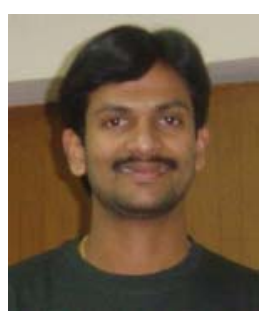

K. Vishnu Vardhan was born in Andhra Pradesh, India. He received the undergraduate degree in Electronics and Communication Engineering from Pondicherry University, Pondicherry, India, in 2005. He received the postgraduate degree in Telecommunication Engineering from Indian Institute of Science, Bangalore, India, in 2007. Since July 2007, he has been with Cisco Systems (India) Private Limited, Bangalore, India. His research interests include multiuser detection and low-complexity detectors for CDMA and MIMO systems.

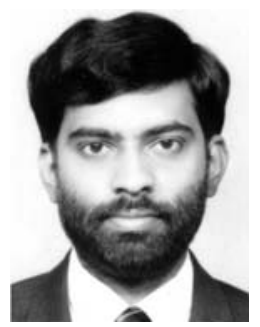

A. Chockalingam received the B.E. (Honors) degree in Electronics and Communication Engineering from the P. S. G. College of Technology, Coimbatore, India, in 1984, the M.Tech. degree with specialization in satellite communications from the Indian Institute of Technology, Kharagpur, India, in 1985, and the Ph.D. degree in Electrical Communication Engineering (ECE) from the Indian Institute of Science (IISc), Bangalore, India, in 1993. During 1986 to 1993 , he worked with the Transmission R \& D division of the Indian Telephone Industries Limited, Bangalore. From December 1993 to May 1996, he was a Postdoctoral Fellow and an Assistant Project Scientist at the Department of Electrical and Computer Engineering, University of California, San Diego (UCSD). From May 1996 to December 1998, he served Qualcomm, Inc., San Diego, CA, as a Staff Engineer/Manager in the systems engineering group. In December 1998, he joined the faculty of the Department of ECE, IISc, Bangalore, India, where he is an Associate Professor, working in the area of wireless communications and networking. He was a visiting faculty to UCSD during summers of 1999 2002. He is a recipient of the Swarnajayanti Fellowship from the Department of Science and Technology, Government of India. He served as an Associate Editor of the IEEE TRANSACTIONS ON VEHICULAR TECHNOLOGY from May 2003 to April 2007. He currently serves as an Editor of the IEEE TRANSACTIONS ON WiRELESS COMMUNiCATIONS. He is also a Guest Editor for the IEEE JOURNAL ON SELECTED AREAS IN COMMUNICATIONS Special Issue on Multiuser Detection for Advanced Communication Systems and Networks. He is a Fellow of the Indian National Academy of Engineering.
Laurence B. Milstein (S66, M68, SM77, F85) received the B.E.E. degree from the City College of New York, New York, NY, in 1964, and the M.S. and $\mathrm{Ph} . \mathrm{D}$. degrees in electrical engineering from the Polytechnic Institute of Brooklyn, Brooklyn, NY, in 1966 and 1968, respectively.

From 1968 to 1974, he was with the Space and Communications Group of Hughes Aircraft Company, and from 1974 to 1976, he was a member of the Department of Electrical and Systems Engineering, Rensselaer Polytechnic Institute, Troy, NY. Since 1976, he has been with the Department of Electrical and Computer Engineering, University of California at San Diego, La Jolla, where he is the Ericsson Professor of Wireless Communications and former Department Chairman, working in the area of digital communication theory with special emphasis on spread-spectrum communication systems. He has also been a consultant to both government and industry in the areas of radar and communications.

Dr. Milstein was an Associate Editor for Communication Theory for the IEEE Transactions on Communications, an Associate Editor for Book Reviews for the IEEE TRANSACTIONS ON INFORMATION THEORY, an Associate Technical Editor for the IEEE COMMUNICATIONS MAGAZINE, and the Editor-in-Chief of the IEEE JOURNAL ON SELECTED AREAS IN Communications. He was the Vice President for Technical Affairs in 1990 and 1991 of the IEEE Communications Society, and is a former Chair of the IEEE Fellows Selection Committee. He is a recipient of the 1998 Military Communications Conference Long Term Technical Achievement Award, an Academic Senate 1999 UCSD Distinguished Teaching Award, an IEEE Third Millennium Medal in 2000, the 2000 IEEE Communication Society Armstrong Technical Achievement Award, and the 2002 MILCOM Fred Ellersick Award. 